\title{
Stimulation of sexual behavior in the male rat by galanin-like peptide
}

\author{
G.S. Fraley ${ }^{\mathrm{a}}$, S.E. Thomas-Smith ${ }^{\mathrm{a}}$, B.V. Acohido ${ }^{\mathrm{b}}$, R.A. Steiner ${ }^{\mathrm{a}, \mathrm{b}, \mathrm{c}}$, D.K. Clifton ${ }^{\mathrm{b}, *}$ \\ ${ }^{\mathrm{a}}$ Department of Physiology and Biophysics, University of Washington, Seattle, WA 98195-6460, USA \\ ${ }^{\mathrm{b}}$ Department of Obstetrics and Gynecology, University of Washington School of Medicine, Seattle, WA 98195-6460, USA \\ ${ }^{\mathrm{c}}$ Department of Biology, University of Washington, Seattle, WA 98195-6460, USA \\ Received 4 February 2004; revised 12 April 2004; accepted 14 April 2004 \\ Available online 10 August 2004
}

\begin{abstract}
Galanin-like peptide (GALP) is a recently described neuropeptide, which shares a partial sequence identity with galanin but is derived from a separate gene. Central injections of GALP stimulate the secretion of gonadotropin-releasing hormone (GnRH) and luteinizing hormone (LH) and induce the expression of Fos in several brain areas known to regulate male sexual behavior in the rat. We postulated that GALP may also stimulate sexual behavior in concert with its stimulatory effect on the hypothalamic-pituitary-gonadal (HPG) axis. To test this hypothesis, we administered GALP, galanin, or the vehicle (artificial cerebrospinal fluid, aCSF) alone to sexually experienced male rats and assessed the effects of these agents on sexual behavior. We observed that compared to aCSF alone, GALP significantly increased all aspects of male-typical sexual behavior, whereas galanin inhibited all of these same behaviors. To examine whether the stimulatory effects of GALP on sexual behavior were mediated by GALP's stimulatory effects on the HPG axis, we castrated the same male rats and repeated the behavioral experiment. We found that GALP maintained its inductive action on male-typical sexual behaviors in the castrated animals, suggesting that the effects of GALP on sexual behavior are not the result of GALP's ability to stimulate testosterone secretion. These observations suggest that GALP neurons are part of the hypothalamic circuitry controlling sexual behavior in the male rat.
\end{abstract}

(C) 2004 Elsevier Inc. All rights reserved.

Keywords: Galanin; Penile erection; Arcuate nucleus

\section{Introduction}

Galanin-like peptide (GALP) is a neuropeptide comprising 60 amino acids. GALP shares a partial amino acid sequence identity with galanin and binds to several galanin receptor subtypes; however, GALP is derived from its own unique gene, distinct from galanin (Ohtaki et al., 1999). GALP is expressed in the brain, primarily in the hypothalamic arcuate nucleus (ARC), where it is regulated by various metabolic signals, including leptin, insulin, and metabolic fuels (Fraley et al., 2003a, 2004; Juréus et al., 2000, 2001; Kumano et al., 2003). Centrally administered GALP has marked effects on feeding and body weight in the

\footnotetext{
* Corresponding author. Department of Obstetrics and Gynecology, University of Washington School of Medicine, PO Box 356460, Seattle, WA 98195-6460. Fax: +1 2065433915.

E-mail address: don@u.washington.edu (D.K. Clifton).
}

rat and mouse (Gundlach, 2002; Krasnow et al., 2003; Lawrence et al., 2002; Matsumoto et al., 2002; Seth et al., 2002, 2003a,b), and it activates the sympathetic nervous system in the mouse (Hansen et al., 2003). In addition, GALP has a direct stimulatory action on gonadotropinreleasing hormone $(\mathrm{GnRH})$ mediated luteinizing hormone (LH) secretion (Cunningham et al., 2002a,b; Gundlach, 2002; Krasnow et al., 2003; Matsumoto et al., 2001; Shen et al., 2002). Taken together, these observations suggest that GALP neurons play a role in relaying humoral signals that reflect the status of metabolic reserves to the hypothalamicpituitary-gonadal (HPG) axis.

During the conduct of studies designed to evaluate the effects of GALP on feeding, body weight, and gonadotropin secretion in male rats, we noticed stereotypical signs of sexual arousal (e.g., considerable yawning and genital grooming), suggesting that GALP might influence reproductive behavior. Furthermore, we had previously shown 
that intracerebroventricular (ICV) injection of GALP induces the expression of Fos in regions of the hypothalamus known to regulate sexual behavior-including the bed nucleus of the stria terminalis $(\mathrm{BnST})$, the medial amygdala (mAg) and most notably, the medial preoptic area (mPOA) (Fraley et al., 2003b; Lawrence et al., 2003). Also, GALP fibers project to these same brain regions (Takatsu et al., 2001). These observations led us to postulate that in addition to activating the HPG axis, GALP also stimulates sexual behavior. To test this hypothesis, we examined the effects of centrally administered GALP (as well as galanin) on sexual behavior in sexually experienced, adult male rats and observed that GALP stimulated all aspects of male-typical sexual behavior compared to vehicle-treated controls in both intact and castrated animals. Our results demonstrate that GALP stimulates sexual behaviors in the male rat and that this inductive action in males occurs in the absence of the secretory products of the testis.

\section{Materials and methods}

\section{Animals}

Adult male (300-320 g) and female (280-300 g) Sprague-Dawley rats were purchased from Charles River Laboratories Inc. (Wilmington, MA). Animals were housed in individual cages and given access to standard rodent chow and water ad libitum. The animals were maintained on a 12:12 light/dark cycle, with lights on at 06:00 h. All experiments were carried out between 08:00 and 12:00 h. Animals were given three bouts of sexual experience before beginning the experiments. All behavioral tests were videotaped for detailed analyses by individuals unaware of the treatment. All procedures were approved by the Animal Care Committee of the School of Medicine at the University of Washington, in accordance with the NIH Guide for Care and Use of Laboratory Animals.

\section{Lateral ventricle cannulations}

Since GALP has the potential to act at several hypothalamic sites, these initial studies utilized cannulas placed into the lateral ventricle. Rats were anesthetized with a ketamine cocktail $(100 \mathrm{mg} / \mathrm{ml}$ ketamine, $20 \mathrm{mg} / \mathrm{ml}$ xylazine, $10 \mathrm{mg} / \mathrm{ml}$ acepromazine, 5.0:2.5:1.0 ratio, respectively) and placed in a stereotaxic frame. A stainless steel 26-gauge cannula (Plastics One, Roanoke, VA) was inserted into the lateral ventricle $(-1.0 \mathrm{~mm}$ midline, 1.0 $\mathrm{mm}$ posterior to Bregma, $\sim 2.5 \mathrm{~mm}$ ventral to dura mater). After surgery, the animals were allowed to recover for 1 week, during which time they were handled daily. All compounds were administered in a volume of $3 \mu \mathrm{l}$ by a 10 $\mu l$ Hamilton syringe (Hamilton Inc., Reno, NV) attached to polyethylene tubing (id: $0.58 \mathrm{~mm}$, od: $0.96 \mathrm{~mm}$ ) and a 33-gauge stainless steel injection cannula. Cannula placement was verified at the end of the experiment by injecting $1 \mu \mathrm{l}$ of blue food-coloring dye before sacrifice and inspecting coronal brain slices for proper location of the dye.

\section{Experimental design}

Experiment 1: effects of ICV GALP on sexual behavior in intact male rats

Intact, sexually experienced male rats $(n=10)$ were given an ICV injection of aCSF, $5.0 \mathrm{nmol}$ galanin (American Peptides), or $5.0 \mathrm{nmol}$ GALP (a gift from Amgen Inc., Thousand Oaks, CA). The dose of GALP was chosen because it has been previously shown to stimulate LH secretion and to induce Fos expression within the mPOA in male rats (Fraley et al., 2003b; Krasnow et al., 2003); galanin was given in an equimolar dose for comparison. Injections were performed so that each rat received all of the treatments. Subsequent injections were carried out at least $72 \mathrm{~h}$ apart. The order of treatments and the testing order were randomized.

Within 5 min following the injection, the rats were placed into a testing arena (polyethylene housing cage) with a sexually experienced steroid-primed female rat $[10 \mu \mathrm{g}$ estradiol benzoate in $0.1 \mathrm{ml}$ safflower oil injected subcutaneously (sc) $48 \mathrm{~h}$ before testing and $500 \mu \mathrm{g}$ progesterone in $0.1 \mathrm{ml}$ safflower oil injected subcutaneously $2 \mathrm{~h}$ before testing]. The males were scored for the number of mounts, intromissions, and ejaculations and the latency to these behaviors in a 20-min test. A mount was recorded if the male approached the female from the rear and placed both forelegs firmly on her flanks and locked his hips (pelvic thrust alone). An intromission was recorded if the foregoing behavior was noted with the addition of a clear pelvic thrust and penile insertion into the vagina, followed by genital grooming.

Experiment 2: effects of ICV GALP on sexual behavior in castrated male rats

To determine whether the effects of GALP on male sexual behavior were mediated indirectly by GALP's stimulatory effect on testosterone secretion, we tested the ability of GALP to induce sexual behavior after castration. The same group of male rats from Experiment 1 were castrated and allowed to recover for 7-10 days. At this time, the experimental design and analyses described for Experiment 1 were repeated.

\section{Statistical analyses}

Analyses of behavioral data were performed with a Wilcoxon Signed Rank Analysis. All analyses were 
performed with GB-Stat Statistical Software (General Dynamics, Inc., Bethesda, MD) for the Macintosh. A $P<$ 0.05 was considered significant.

\section{Results}

Experiment 1: effects of ICV GALP on sexual behavior in intact male rats

Central injection of $5.0 \mathrm{nmol}$ GALP elicited significant increases in the number of mounts $(P<0.01)$, intromissions $(P<0.01)$, and ejaculations $(P<0.001)$, compared to aCSF-injected rats (see Fig. 1). GALP also produced a
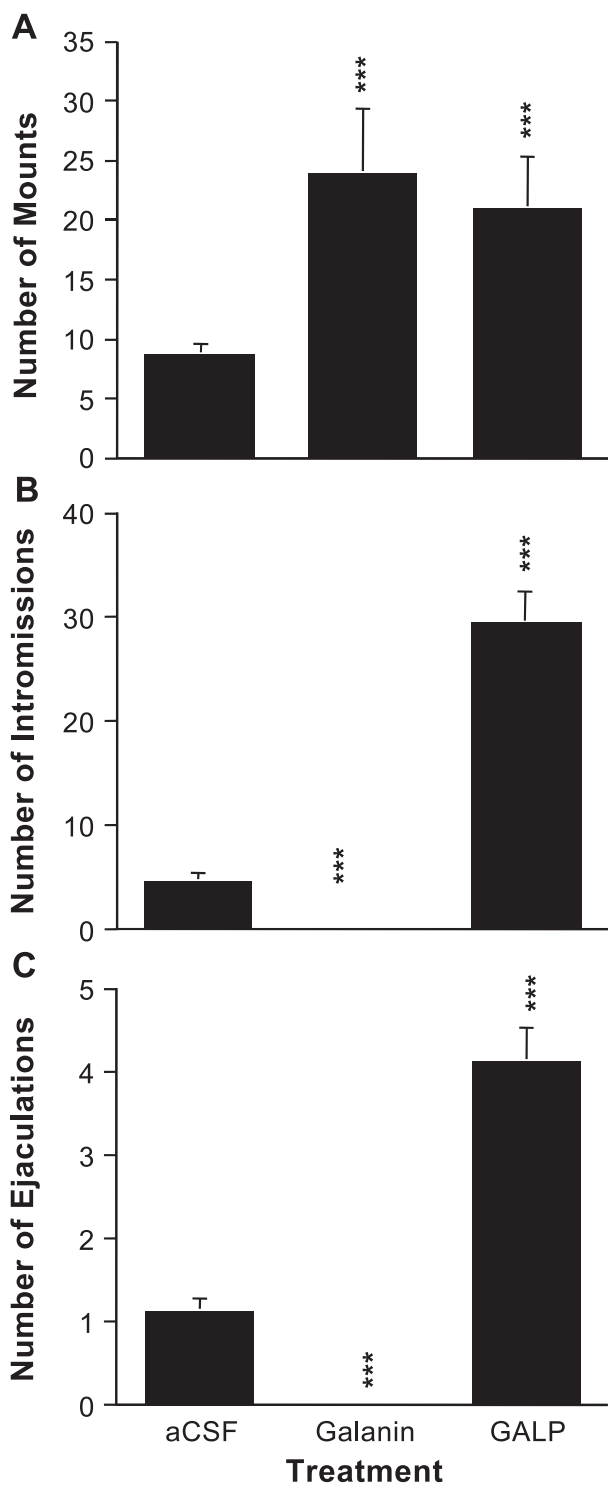

Fig. 1. Effects of ICV GALP, galanin, or aCSF on sexual behaviors in intact male rats. GALP facilitated the number of (A) mounts, (B) intromissions, and $(C)$ ejaculations. Although galanin increased the number of mounts, it inhibited intromissions and ejaculations. ${ }^{* * *} P<0.001$, compared to vehicle-only (aCSF) controls, $n=10$ per group. significant decrease in the latency to all of these behaviors (Fig. 2, $P<0.001$ ), again compared to either the vehicle alone or galanin. ICV galanin also significantly increased the number of mounts compared to aCSF-injected animals (Fig. 1, $P<0.01$ ). Conversely, ICV galanin nearly abolished intromission and ejaculatory behaviors. There was no significant difference in the ratio of intromissions:ejaculations following ICV GALP administration (data not shown). Although there was an increase in the number of mounts per intromission in the GALP-treated rats, this effect is difficult to interpret because the male's GALPinduced sexual voracity appeared to make the females less proceptive (data not shown). When the animals were given aCSF, their sexual behavior was not significantly different from the way they behaved at the end of the preoperative training bouts $(P>0.05$; data not shown), suggesting that the injection of aCSF did not influence sexual performance.

Experiment 2: effects of ICV GALP on sexual behavior in castrated male rats

Following castration, animals treated with aCSF alone showed a reduction in the number of sexual behaviors relative to the intact state (compare Figs. 1 and 3). However, ICV GALP elicited a significant increase in the number of mounts $(P<0.001)$ and intromissions $(P<$ $0.001)$ and a significant reduction in the latency to these two behaviors $(P<0.001)$ compared to either the vehicle alone or galanin (see Figs. 3 and 4). Galanin again significantly reduced the number of mounts $(P<0.05)$, but had no discernible effect on intromissions compared to aCSF controls. No ejaculations were observed following any of the injections.

\section{Discussion}

We have shown that centrally administered GALP acutely stimulates sexual behavior in the male rat. GALP increased the number of mounts, intromissions, and ejaculations, while decreasing the latency to all of these behaviors. Precisely where in the brain GALP acts to facilitate male sexual behaviors is currently unknown. However, previous studies from our laboratory and others have shown that ICV GALP induces the expression of the immediate early gene product Fos in the mPOA, BnST, and $\mathrm{mAg}$ - areas recognized to play an important role in the regulation of sexual behavior in the male rat (see Paredes and Baum, 1997 for review), and which are also principle targets for projections from GALP neurons (Takatsu et al., 2001). Furthermore, induction of Fos expression in the mPOA and BnST accompanies the activation of male sexual behavior (see Pfaus and Heeb, 1997 for review). Taken together, these observations suggest that the mPOA or BnST are nodal points for mediating GALP's effects on repro- 

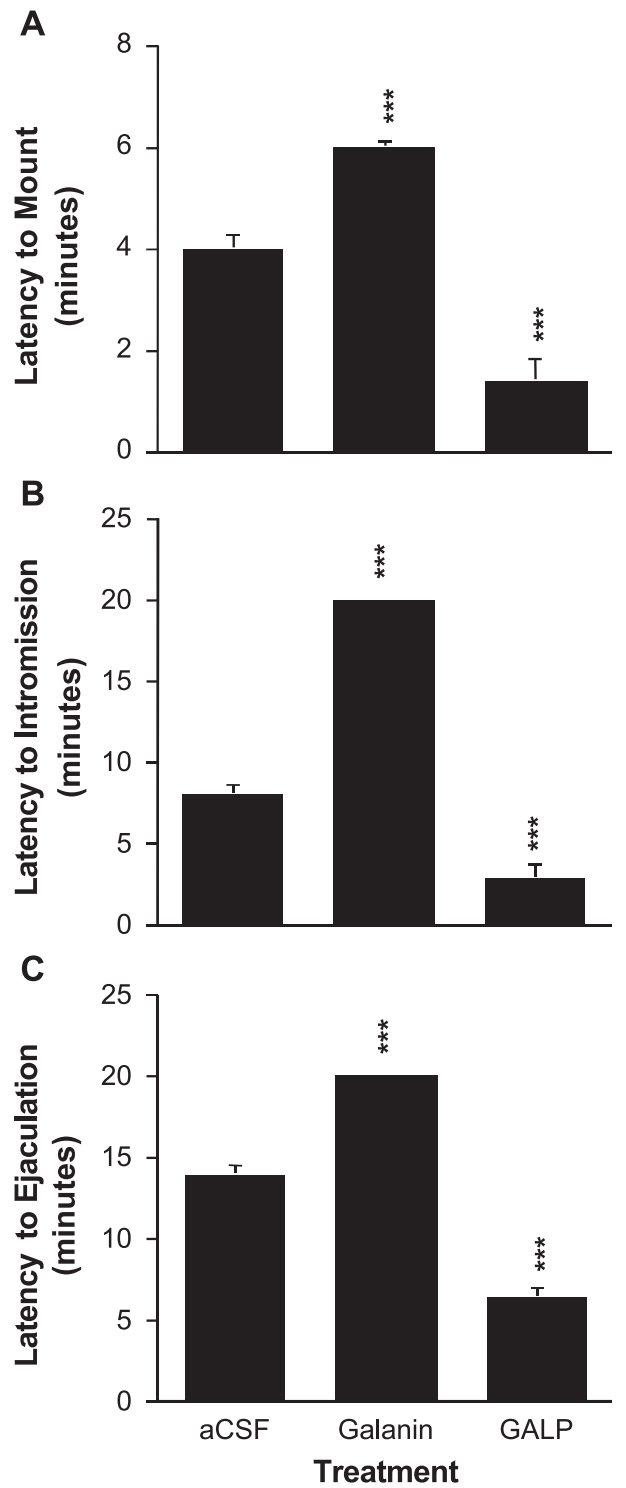

Fig. 2. Effects of ICV GALP, galanin, or aCSF on latencies to male sexual behaviors. GALP decreased and galanin increased the latency to first (A) mount, (B) intromission, and (C) ejaculation. (A 20-min latency indicates the behavior was not observed during the 20 -min test.) $* * * P<0.001$ compared to vehicle-only (aCSF) controls, $n=10$ per group.

ductive behavior in the male rat, although the exact site of action of GALP on male sexual behavior cannot be determined from these experiments.

The effects of GALP administration are reminiscent of those seen following activation of dopaminergic pathways in male rats (see Hull et al., 1999 for review; Melis and Argiolas, 1995). On one hand, it seems plausible that GALP acts through dopamine neurons to facilitate sexual behavior. Although connections between GALP- and dopamineexpressing neurons have not been established, a preliminary report from Kageyama et al. (2003) suggests that GALP injections induce Fos expression in tyrosine hydroxylaseexpressing (dopamine) neurons located in the ARC. Dopamine neurons in the ARC have not been directly implicated in the regulation of sexual behavior, but it is conceivable that further investigations will reveal that other populations of dopaminergic neurons are also activated by GALP (Melis and Argiolas, 1995). On the other hand, it is also possible that dopamine neurons could be acting through GALP neurons to regulate sexual behavior. Again, interactions between dopamine and GALP neurons have yet to be investigated. Nevertheless, the fact that dopamine is itself thought to act directly on the mPOA argues against the need for GALP neurons to mediate the effects of dopamine (unless dopamine acts presynaptically on GALP terminals in the mPOA) (Hull et al., 1999). A third possibility is that GALP acts independently but in parallel with dopamine in the mPOA (or BnST or $\mathrm{mAg}$ ) to facilitate male sexual behavior. This concept is consonant with the presence of GALP-containing fibers in the mPOA and a direct action of dopamine on the mPOA, and suggests that both GALP and dopamine innervate the same population of neurons in the $\mathrm{mPOA}$ to regulate reproductive behavior.

GALP stimulates the GnRH-mediated release of LH, which increases testosterone secretion (Matsumoto et al., 2001; Seth et al., 2003a,b). Since testosterone arouses male

\section{A}

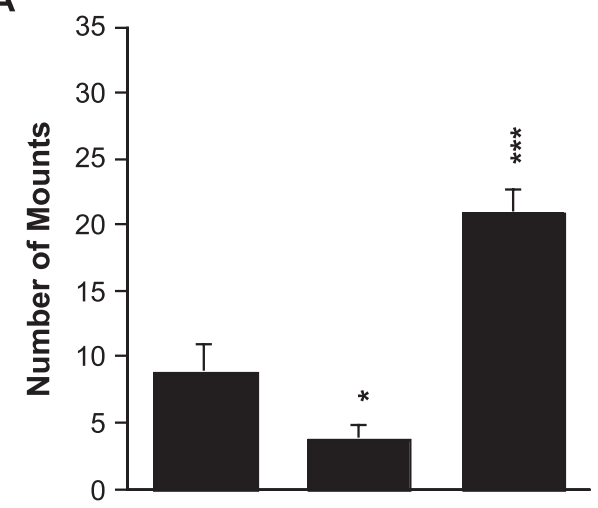

B

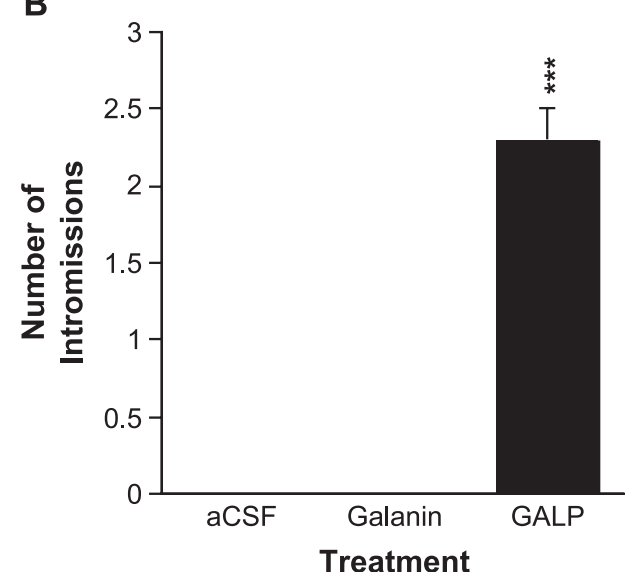

Fig. 3. Effects of ICV GALP, galanin, or aCSF on sexual behaviors in castrated male rats. GALP significantly increased the number of (A) mounts and (B) intromissions, while galanin significantly decreased the number of mounts in the absence of testosterone. No ejaculations were observed during the 20 -min test. $* P<0.05$ and $* * * P<0.001$, compared to vehicleonly (aCSF) controls, $n=10$ per group. 
A
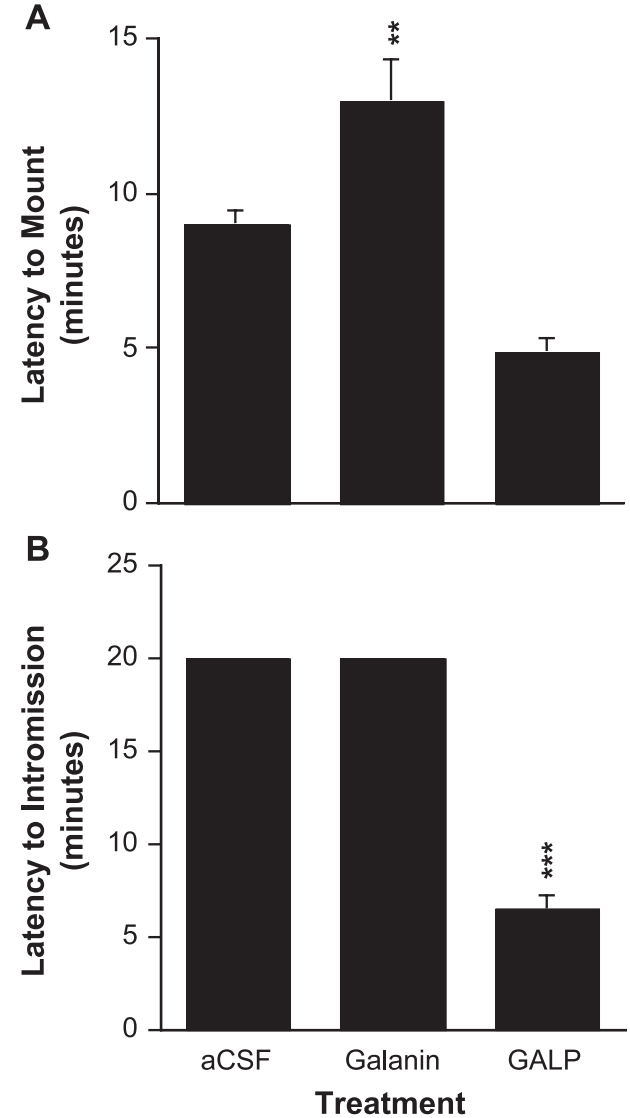

Fig. 4. Effects of ICV GALP, galanin, or aCSF on latencies to sexual behaviors in castrated male rats. GALP significantly decreased the latency to first (A) mount and (B) intromission compared to controls, while galanin had no effect in the absence of testosterone. No ejaculations were observed during this test. (A 20-min latency indicates the behavior was not observed during the 20-min test.) ${ }^{*} P<0.01$ and $* * * P<0.001$, compared to vehicle-only (aCSF) controls, $n=10$ per group.

sexual behavior (Beach and Holz-Tucker, 1949; Damassa et al., 1977), we considered the possibility that the observed effects of GALP on sexual behavior in the intact male rat was attributable to increased testosterone secretion. We tested this hypothesis by investigating the effects of GALP in castrated males. The results of this study indicate that GALP's stimulatory effect on sexual behavior occurs in the absence of testosterone, since GALP stimulated mounting behavior and intromissions in castrated animals. However, the response to GALP was reduced in castrated animals compared to intact controls, indicating that testosterone acts permissively to support male sexual behavior-notwithstanding GALP's ability to stimulate sexual behavior acutely in both intact and castrated animals. Any interpretation of the attenuated response to GALP in castrated rats is confounded by the fact that castration also caused an increase in mounting latency and completely abolished intromissions and ejaculations in control (non GALPtreated) animals. Treatment with GALP partially reversed this decline. However, GALP did not fully restore either mounting latency or intromissions in the castrated animals, and it failed to restore ejaculations, perhaps because there are too few intromissions. Nevertheless, GALP's ability to partially restore sexual behavior in castrated rats is consistent with the concept that testosterone does not wholly mediate the effects of GALP on these behaviors.

Since the expression of GALP mRNA is unaffected by sex steroids in either the rat or macaque (Cunningham et al., 2003), it seems unlikely that GALP mediates the effects of gonadal steroids on reproductive behavior. However, the expression of GALP is altered by a variety of circulating metabolic signals, including leptin, insulin, free fatty acids, and glucose; furthermore, GALP-expressing neurons in the ARC coexpress the leptin receptor and insulin receptor substrate-2 (Juréus et al., 2000; Fraley et al., 2003a). Because metabolic abnormalities associated with diabetes and fasting have been shown to influence both male and female sexual behavior (Hassan et al., 1993; Karkanias et al., 1997; Steger, 1990; Steger et al., 1989; Wade and Schneider, 1992), GALP is a plausible candidate for transmitting metabolic information to neuronal circuits involved in regulating reproductive function, including behavior. If this were the case, one might expect GALP to function in a permissive fashion, allowing sexual behavior to proceed when nutrition is adequate and restraining such behavior when conditions are unfavorable. Nevertheless, the fact that GALP was found to stimulate sexual behavior in well-fed animals suggests that GALP plays more than a permissive role.

GALP binds in vitro to several galanin receptor subtypes (at least to GALR1 and GALR2), and we thought it was important to determine whether the effects of GALP on sexual behavior might be mediated by one or more of the galanin receptors. We argued that if this were the case, galanin should also activate these receptors and thus have stimulatory effects on these behaviors-notwithstanding some published evidence to the contrary. Reports on the effects of galanin on sexual behavior have been equivocal. Galanin has been shown to stimulate sexual behavior in the male either when injected into the mPOA of the rat or when given ICV into the mouse (Benelli et al., 2002; Bloch et al., 1993); however, Poggioli et al. report that galanin inhibits sexual behavior when it is injected ICV into the male rat. Similarly, Benelli et al. (1994) showed an increase in male rat sexual behavior following a central injection of a galanin receptor antagonist. Our demonstration that galanin stimulates mounting behavior but inhibits intromissions and ejaculations in the male rat corroborates the work of both Poggioli et al. (1992) and Benelli et al. One possible explanation for the discrepancies among the reported effects of galanin administration via different routes is that galanin injected into the mPOA activates stimulatory pathways in that region that are normally innervated by GALP projections. It is also possible that ICV galanin inhibits the male rat's ability to achieve an erection, thus eliciting an increase in mounting behavior (that does not require an erection) but inhibiting sex behavior overall. However, regardless of any 
putative role for galanin in regulating sexual behavior, the observation that GALP and galanin - when administered in a similar manner - had strikingly divergent effects on male sexual behavior argues that exogenously administered GALP does not stimulate sexual behavior in the rat by interacting with galanin receptors. Taken together with our previous observation that galanin and GALP activate Fos expression in different populations of hypothalamic neurons (Fraley et al., 2003b), these results suggest that GALP does not act via galanin receptors (GALR1 or GALR2), but instead utilizes its own unique receptor(s).

Our experiments were performed at the onset of the rats' light cycle. Since rats are nocturnal, this time frame may have resulted in a blunted magnitude in the measured behaviors. However, mating behavior in rats has been shown to occur both nocturnally and diurnally, and the levels of sexual behaviors in this experiment are similar to that reported previously (Blanchong et al., 1999; Logan and Leavitt, 1992). Similarly, Stefanick (1983) reported no significant time-of-day effects on reflexive erections. Thus, we think it is unlikely that we would have obtained different outcomes if these same experiments had been performed during the dark phase, although it is conceivable that a different magnitude of behaviors might have been observed.

In summary, we have shown that centrally administered GALP induces sexual behavior in the male rat. This effect of GALP on sexual behavior extends previous observations demonstrating that GALP is involved in the regulation of gonadotropin secretion, feeding, and body weight. The fact that GALP mRNA-expressing neurons are regulated by metabolic hormones and that GALP stimulates both the HPG axis as well as sexual behavior highlights the potential importance of GALP in the integration of metabolism and reproduction. Finally, based on these observations we infer that sexual dysfunction associated with metabolic diseases, such as diabetes, could be mediated by alterations in the activity of GALP neurons and further that therapeutic strategies that involve GALP, its analogues, or its putative receptor might prove useful in treating these disorders.

\section{Acknowledgments}

We are grateful to Amgen Inc. for their kind gift of the GALP. We also thank Drs. Susan M. Fraley, Jeremy Smith, Michelle Gottsch, as well as Ms. Stephanie Krasnow for their critical comments and assistance in the production of this manuscript.

\section{References}

Beach, F.A., Holz-Tucker, A.M., 1949. Effects of different concentrations of androgen upon sexual behavior in castrated male rats. J. Comp. Physiol. Psychol. 42, 433-453.
Benelli, A., Arletti, R., Bertolini, A., Menozzi, B., Basaglia, R., Poggioli, R., 1994. Galantide stimulates sexual behaviour in male rats. Eur. J. Pharmacol. 260 (2-3), 279-282.

Benelli, A., Bertolini, A., Zoli, M., Leo, G., Filaferro, M., Saltini, S., Genedani, S., 2002. Pharmacological manipulation of brain galaninergic system and sexual behavior in male mice. Psychopharmacology (Berl.) 160 (3), 325-330.

Blanchong, J.A., McElhinny, T.L., Mahoney, M.M., Smale, L., 1999. Nocturnal and diurnal rhythms in the unstriped Nile rat, Arvicanthis niloticus. J. Biol. Rhythms 14 (5), 364-377.

Bloch, G.J., Butler, P.C., Kohlert, J.G., Bloch, D.A., 1993. Microinjection of galanin into the medial preoptic nucleus facilitates copulatory behavior in the male rat. Physiol. Behav. 54 (4), 615-624.

Cunningham, M.J., Krasnow, S.M., Jureus, A., Li, D., Carlson, A.E., Teklemichael, D.N., Clifton, D.K., Steiner, R.A., 2002a. Galanin-like peptide (GALP) as a potential regulator of pituitary hormone secretion. Soc. Neurosci.

Cunningham, M.J., Scarlett, J.M., Steiner, R.A., 2002b. Cloning and distribution of galanin-like peptide mRNA in the hypothalamus and pituitary of the macaque. Endocrinology 143 (3), 755-763.

Cunningham, M.J., Krasnow, S.M., Gevers, E.F., Chen, P., Thompson, C.K., Robinson, I.C.A.F., Smith, M.S., Clifton, D.K., Steiner, R.A., 2003. Regulation of galanin-like peptide gene expression by pituitary hormones and their downstream targets. J. Neuroendocrinol. $15,1-9$.

Damassa, D.A., Smith, E.R., Tennent, B., Davidson, J.M., 1977. The relationship between circulating testosterone levels and male sexual behavior in rats. Horm. Behav. 8 (3), 275-286.

Fraley, G.S., Scarlett, J.M., Teklemichael, D.N., Shimada, I., Baumgartner, J.W., Acohido, B., Clifton, D.K., Steiner, R.A., 2003a. Regulation of galanin-like peptide gene expression by insulin and metabolic fuels in the hypothalamus of the rat. Endocrine Society Annual Meeting.

Fraley, G.S., Shimada, I., Baumgartner, J.W., Clifton, D.K., Steiner, R.A., 2003b. Differential patterns of Fos induction in the hypothalamus of the rat following central injections of galanin-like peptide and galanin. Endocrinology 144 (4), 1143-1146.

Fraley, G.S., Scarlett, J.M., Shimada, I., Teklemichael, D.N., Acohido, B.V., Clifton, D.K., Steiner, R.A., 2004. The effects of diabetes and insulin on the expression of galanin-like peptide (GALP) in the hypothalamus of the rat. Diabetes 53, 1237-1242.

Gundlach, A.L., 2002. Galanin/GALP and galanin receptors: role in central control of feeding, body weight/obesity and reproduction? Eur. J. Pharmacol. 440 (2-3), 255-268.

Hansen, K.R., Krasnow, S.M., Nolan, M.A., Fraley, G.S., Baumgartner, J.W., Clifton, D.K., Steiner, R.A., 2003. Activation of the sympathetic nervous system by galanin-like peptide-A possible link between leptin and metabolism. Endocrinology 144 (11), 4709-4717.

Hassan, A.A., Hassouna, M.M., Taketo, T., Gagnon, C., Elhilali, M.M., 1993. The effect of diabetes on sexual behavior and reproductive tract function in male rats. J. Urol. 149 (1), 148-154.

Hull, E.M., Lorrain, D.S., Du, J., Matuszewich, L., Lumley, L.A., Putnam, S.K., Moses, J., 1999. Hormone-neurotransmitter interactions in the control of sexual behavior. Behav. Brain Res. 105 (1), 105-116.

Juréus, A., Cunningham, M.J., McClain, M.E., Clifton, D.K., Steiner, R.A., 2000. Galanin-like peptide (GALP) is a target for regulation by leptin in the hypothalamus of the rat. Endocrinology 141 (7), 2703-2706.

Juréus, A., Cunningham, M.J., Li, D., Johnson, L.L., Krasnow, S.M., Teklemichael, D.N., Clifton, D.K., Steiner, R.A., 2001. Distribution and regulation of galanin-like peptide (GALP) in the hypothalamus of the mouse. Endocrinology 142 (12), 5140-5144.

Kageyama, H., Takenoya, F., Funahashi, H., Inoue, K., Matsumoto, H., Ohtaki, T., Inoue, S., Shioda, S., 2003. Galanin-like peptide mediates feeding regulation through tyrosine hydroxylase containing neurons in the hypothalamus. Soc. Neurosci.

Karkanias, G.B., Morales, J.C., Li, C.S., 1997. Deficits in reproductive behavior in diabetic female rats are due to hypoinsulinemia rather than hyperglycemia. Horm. Behav. 32 (1), 19-29. 
Krasnow, S.M., Fraley, G.S., Schuh, S.M., Baumgartner, J.W., Clifton, D.K., Steiner, R.A., 2003. A role for galanin-like peptide (GALP) in the integration of feeding, body weight regulation and reproduction in the mouse. Endocrinology 144 (3), 813-822.

Kumano, S., Matsumoto, H., Takatsu, Y., Noguchi, J., Kitada, C., Ohtaki, T., 2003. Changes in hypothalamic expression levels of galanin-like peptide in rat and mouse models support that it is a leptin-target peptide. Endocrinology 144 (6), 2634-2643.

Lawrence, C.B., Baudoin, F.M., Luckman, S.M., 2002. Centrally administered galanin-like peptide modifies food intake in the rat: a comparison with galanin. J. Neuroendocrinol. 14 (11), 853-860.

Lawrence, C.B., Williams, T., Luckman, S.M., 2003. Intracerebroventrilcular galanin-like peptide induces different brain activation compared with galanin. Endocrinology 144 (9), 3977-3984.

Logan, F.A., Leavitt, F., 1992. Sexual free behavior in male rats (Rattus norvegicus). J. Comp. Psychol. 106 (1), 37-42.

Matsumoto, H., Noguchi, J., Takatsu, Y., Horikoshi, Y., Kumano, S., Ohtaki, T., Kitada, C., Itoh, T., Onda, H., Nishimura, O., Fujino, M., 2001. Stimulation effect of galanin-like peptide (GALP) on luteinizing hormone-releasing hormone-mediated luteinizing hormone (LH) secretion in male rats. Endocrinology 142 (8), 3693-3696.

Matsumoto, Y., Watanabe, T., Adachi, Y., Itoh, T., Ohtaki, T., Onda, H., Kurokawa, T., Nishimura, O., Fujino, M., 2002. Galanin-like peptide stimulates food intake in the rat. Neurosci. Lett. 322 (1), 67-69.

Melis, M.R., Argiolas, A., 1995. Dopamine and sexual behavior. Neurosci. Biobehav. Rev. 19 (1), 19-38.

Ohtaki, T., Kumano, S., Ishibashi, Y., Ogi, K., Matsui, H., Harada, M., Kitada, C., Kurokawa, T., Onda, H., Fujino, M., 1999. Isolation and cDNA cloning of a novel galanin-like peptide (GALP) from porcine hypothalamus. J. Biol. Chem. 274 (52), 37041-37045.

Paredes, R.G., Baum, M.J., 1997. Role of the medial preoptic area/anterior hypothalamus in the control of masculine sexual behavior. Annu. Rev. Sex Res. 8, 68-101.
Pfaus, J.G., Heeb, M.M., 1997. Implications of immediate-early gene induction in the brain following sexual stimulation of female and male rodents. Brain Res. Bull. 44 (4), 397-407.

Poggioli, R., Rasori, E., Bertolini, A., 1992. Galanin inhibits sexual behavior in male rats. Eur. J. Pharmacol. 213 (1), 87-90.

Seth, A., Stanley, S.A., Dhillo, W., Murphy, K.G., Bloom, S.R., 2002. The novel hypothalamic peptide galanin-like peptide (GALP) stimulates food intake in male Wistar rats. Endocr. Abstr. 2 (OC17).

Seth, A., Stanley, S., Dhillo, W., Murphy, K., Ghatei, M., Bloom, S., 2003a. Effects of galanin-like peptide on food intake and the hypothalamopituitary-thyroid axis. Neuroendocrinology 77 (2), 125-131.

Seth, A., Stanley, S., Jethwa, P., Gardiner, J., Ghatei, M., Bloom, S., 2003b. Galanin-like peptide stimulates the release of gonadotropin-releasing hormone in vitro and may mediate the effects of leptin on the hypothalamo-pituitary-gonadal axis. Endocrinology.

Shen, J., Balourdos, G., O’Donnell, L., Gundlach, A.L., 2002. Pubertal induction of galanin-like peptide (GALP) expression in the hypothalamus and testis: links with LHRH signaling? Proc. Soc. Neurosci.

Stefanick, M.L., 1983. The circadian patterns of spontaneous seminal emission, sexual activity and penile reflexes in the rat. Physiol. Behav. 31 (6), 737-743.

Steger, R.W., 1990. Testosterone replacement fails to reverse the adverse effects of streptozotocin-induced diabetes on sexual behavior in the male rat. Pharmacol. Biochem. Behav. 35 (3), 577-582.

Steger, R.W., Amador, A., Lam, E., Rathert, J., Weis, J., Smith, M.S., 1989. Streptozotocin-induced deficits in sex behavior and neuroendocrine function in male rats. Endocrinology 124 (4), 1737-1743.

Takatsu, Y., Matsumoto, H., Ohtaki, T., Kumano, S., Kitada, C., Onda, H., Nishimura, O., Fujino, M., 2001. Distribution of galanin-like peptide in the rat brain. Endocrinology 142 (4), 1626-1634.

Wade, G.N., Schneider, J.E., 1992. Metabolic fuels and reproduction in female mammals. Neurosci. Biobehav. Rev. 16 (2), 235-272. 